\title{
Tolerância de Himatanthus sucuuba Wood. (Apocynaceae) ao alagamento na Amazônia Central ${ }^{1}$
}

\author{
Cristiane Ferreira ${ }^{2,5}$, Maria Teresa Fernandez Piedade ${ }^{2}$, Pia Parolin ${ }^{3}$ e Keillah Mara Barbosa ${ }^{4}$
}

Recebido em 08/07/2003. Aceito em 05/11/2004

\begin{abstract}
RESUMO - (Tolerância de Himatanthus sucuuba Wood. (Apocynaceae) ao alagamento na Amazônia Central). Himatanthus sucuuba é uma espécie arbórea de reputado valor fitoterápico, que coloniza regiões de várzea e de terra firme na Amazônia Central. Objetivou-se verificar durante quatro meses, em casa de vegetação, a germinação e o desenvolvimento inicial de plântulas da espécie, oriundas de sementes dos dois diferentes ecossistemas. Não houve diferença significativa em relação ao peso de matéria seca de sementes da várzea e da terra firme. Entretanto, a taxa de germinação da espécie variou de acordo com o substrato e o local de procedência das sementes. No substrato areia+serragem (não-alagado) a germinação da espécie foi alta, independente do ambiente (várzea $94 \%$ e terra firme $91 \%$ ). No substrato constituído apenas por água (alagado), as sementes provenientes da várzea tiveram a maior taxa de germinação ( $96 \%$ ) e de formação de plântulas (84\%), quando relacionadas às da terra firme, que apresentaram taxa de germinação de 64\%, e nenhuma das sementes germinadas formou plântulas. Neste tratamento foi verificado o apodrecimento da radícula poucos dias após sua emissão, indicando que as sementes oriundas da várzea apresentam características adaptativas ao ambiente inundado em relação às da mesma espécie oriundas da terra firme.
\end{abstract}

Palavras-chave: anoxia, hipoxia, dispersão hidrocórica, estabelecimento de plântulas

\begin{abstract}
Tolerance of Himatanthus sucuuba Wood. (Apocynaceae) to the flooding regime in the Central Amazonian). Himatanthus sисииьa is a tree species with known phytotherapic value which colonizes both white-water floodplains (várzea) and upland forests (terra firme) in Central Amazonian. The objective of this study was to compare germination and initial seedling development from seeds collected in both the ecosystems. There was no significant difference between dry seed mass, but in a four month period in a glasshouse in Manaus, Brazil, germination rate differed according to the substrate and origin of the seeds. In the substrate sand + sawdust (nonflooded) germination rate was high, independent of seed origin (várzea $94 \%$ and terra firme $91 \%$ ). In the substrate constituted only by water (flooded), the seeds originating from várzea had a higher germination rate (96\%) and seedling formation (84\%) than those originating from terra firme which had a germination rate of $64 \%$ and none of the seeds formed seedlings. In this treatment, the radicles rot few days after their emission, indicating that the seeds originating from várzea present adaptive characteristics to the floodplain environment which are not present in seeds from terra firme.
\end{abstract}

Key words : anoxia, hypoxia, hydrochoric dispersal, seedlings establishment

\section{Introdução}

$\mathrm{O}$ alagamento do solo tem profundo efeito nos organismos que nele habitam, especialmente nas plantas, por serem sésseis (Piedade et al. 2001). A maioria das sementes de plantas terrestres que possui alta taxa de germinação no solo não germina na água, uma vez que estas perdem rapidamente a viabilidade sob tais condições (Hook 1984; Parolin 2001). Dessa forma, a inundação influencia a germinação de sementes e o desenvolvimento de plântulas. A ativação dos processos fisiológicos necessários para a germinação requer suprimento adequado de oxigênio e o alagamento do solo restringe a disponibilidade de oxigênio para o embrião, impedindo a germinação ou impondo dormência nas sementes de muitas espécies (Kozlowski 1997; Kozlowski \& Pallardy 1997).

Por outro lado, na Amazônia Central, muitas espécies de árvores crescem em áreas de várzea, onde o período de inundação contínua pode durar cerca de 210 dias todos os anos e durante esta fase, o solo é coberto por uma coluna de água que pode chegar, em média, até $10 \mathrm{~m}$ (Junk 1989). Sob tais condições, a maioria das plântulas são completamente alagadas

\footnotetext{
Parte da dissertação de Mestrado do primeiro Autor

Instituto Nacional de Pesquisas da Amazônia, Projeto INPA/Max-Planck, C. Postal 478, CEP 69011-970, Manaus, AM, Brasil

Max Planck Institut for Limnology, Tropical Ecology, P.O. Box 165, 24302 Plön, Germany

Universidade Federal do Paraná, Departamento de Ciências Florestais, Rua Bom Jesus 650, CEP 88035-010, Curitiba, PR, Brasil

Autor para correspondência: crisfer@inpa.gov.br
} 
(Parolin 2000; Piedade et al. 2000).

Devido às características hídricas distintas, existem diferenças entre a flora de várzea e de terra firme e só $18 \%$ das espécies de árvores são comuns aos dois ambientes (Campbell et al. 1986; Piedade et al. 2000). Um exemplo é Himatanthus sucuuba Wood., Apocynaceae de porte arbóreo e de grande interesse para a economia regional, especialmente pelo reputado valor fitoterápico atribuído ao látex da espécie, que é indicado na medicina popular para tratamentos de fraturas, problemas gástricos e como anticancerígeno (Villegas et al. 1997; Ribeiro et al. 1999).

Em terra firme $H$. sucuuba habita áreas que não estão sujeitas a períodos prolongados de saturação de água no solo (Ribeiro et al. 1999). Nos ambientes alagados, sua ocorrência se dá em regiões de várzea baixa, onde o alagamento ininterrupto chega a durar cerca de cinco meses todos os anos (Wittmann et al. 2002). Nos dois locais, a planta é encontrada ocorrendo em áreas abertas nos bordos das florestas. Os frutos são deiscentes, com numerosas sementes elipsóides secas, envoltas por uma ala membranosa circular bem desenvolvida. Esta estrutura reveste inteiramente a semente protegendo e facilitando a dispersão da espécie pelo vento (anemocoria). A polinização é realizada por insetos da família Sphingidae (Plumel 1991; Lorenzi 1998).

Himatanthus sucuuba foi escolhida para ajudar a elucidar, mediante estudos de germinação e formação de plântulas, os mecanismos adaptativos de plantas em ambientes com diferentes pressões ambientais, e estabelecer os limites de tolerância das mesmas ao alagamento. Este estudo visou responder à seguinte pergunta: o pulso de inundação, que anual e regularmente alaga grandes áreas ao longo do rio Solimões/Amazonas, pode induzir, dentro de uma mesma espécie, mudanças detectáveis entre populações habitando o ambiente alagável e o de terra firme, a fim de assegurar a sobrevivência de suas plântulas em ambos os habitats?

\section{Material e métodos}

Frutos coletados na várzea na Ilha da Marchantaria $\left(03^{\circ} 15^{\prime} \mathrm{S} 60^{\circ} 00^{\prime} \mathrm{W}\right)$, e na terra firme, Reserva Florestal Adolpho Ducke (0253' S 59 58 W) foram transportados para o Instituto Nacional de Pesquisas da Amazônia (INPA), Manaus e deixados ao sol para completar a abertura e liberação das sementes. Em seguida, um lote de 100 sementes de cada ambiente (várzea e terra firme) foi separado, em quatro repetições de 25 , para a determinação do peso de matéria seca, adotando-se o método de secagem em estufa a $105 \pm 3{ }^{\circ} \mathrm{C}$, por 24 horas (Brasil 1992).

Foram tomadas medidas biométricas (comprimento, largura e espessura) de 100 sementes por ambiente de origem, com e sem a ala que as envolvia. $\mathrm{O}$ mesmo procedimento foi adotado para a realização dos testes de germinação, com a finalidade de verificar se a remoção da mesma poderia interferir na velocidade da germinação. A retirada da ala foi feita manualmente, deixando totalmente livre a região da micrópila. Em seguida, as sementes foram desinfestadas com hipoclorito de sódio a $10 \%$ da solução comercial, durante 15 minutos, sendo posteriormente lavadas em água corrente. O experimento foi montado em viveiro, onde as sementes foram colocadas para germinar em bandejas de $20 \times 60 \mathrm{~cm}$ à temperatura ambiente (média diária de $28^{\circ} \mathrm{C}$ ) e submetidas a dois diferentes tratamentos, os quais tentaram simular os ambientes naturais da espécie, sendo divididos em: a) não-alagado e b) alagamento. $\mathrm{O}$ tratamento não-alagado foi constituído dos substratos serragem + areia lavada, na proporção volumétrica de 1:1, com regas diárias, enquanto que o tratamento de alagamento foi constituído apenas por uma coluna de água de $5 \mathrm{~cm}$. A água utilizada foi proveniente de poço artesiano localizado nas dependências do INPA. Cada bandeja foi considerada uma amostra e os testes constaram de quatro repetições de 100 sementes para cada tratamento. A protrusão da radícula indicou o fim dos testes de germinação, que nas sementes sem o revestimento alado tiveram duração máxima de 15 dias. Para as sementes intactas, devido à ausência de germinação, os testes tiveram esse período estendido para 30 dias. Depois de germinadas, as sementes permaneceram nos substratos durante 45 dias, quando foi feito o acompanhamento até a formação de plântulas com cerca de $7 \mathrm{~cm}$ alt.

$\mathrm{O}$ delineamento experimental foi inteiramente casualizado. Foi feita análise de variância (ANOVA) e a comparação entre as médias dos fatores estudados foi realizada com base no teste de Tukey, a 5\% de probabilidade.

\section{Resultados}

O tipo de germinação apresentado pelas sementes de $H$. sucuuba, independente do ambiente de origem, se várzea ou terra firme, é epígea, fanerocotiledonar.

A comparação do peso médio de matéria seca das sementes oriundas dos dois diferentes ambientes 
não mostrou diferença significativa ( $p>0,05)$, resultado semelhante ao obtido para as medidas biométricas (Tab. 1).

Durante o período de observação de 30 dias, nenhuma das sementes cujo revestimento alado foi mantido germinou, independente do ambiente e do substrato. Por outro lado, nos lotes de sementes em que foi feita a remoção destes revestimentos, ficando livre a região da micrópila, a germinação máxima durou cerca de 15 dias e foram encontradas variações nas taxas de germinação, relacionadas com o tipo de substrato e o local de procedência das sementes (Fig. 1A).

No tratamento não-alagado, $91 \%$ das sementes oriundas de locais da terra firme e $94 \%$ da várzea germinaram, sendo que essa diferença não foi significativa. Entretanto, no tratamento de alagamento, a germinação foi de $64 \%$ entre as sementes da terra firme e $96 \%$ entre as da várzea, diferença significativa (Fig. 1A). Independente do ambiente de origem, todas as sementes do tratamento não-alagado, que germinaram, produziram plântulas (100\%); enquanto que das sementes dos dois ambientes que germinaram no tratamento de alagamento, apenas as provenientes da várzea (84\%) atingiram essa fase (Fig. 1B). As sementes da terra firme que germinaram na água tiveram longevidade máxima de cinco dias, não chegando a produzir plântulas devido ao apodrecimento das raízes.

\section{Discussão}

A germinação e a formação de plântulas da espécie H. sucuuba variou de acordo com o ambiente de origem das sementes: o número de sementes germinadas da população da várzea, como também o número de plântulas, foi maior em relação à população da terra firme, para o tratamento de alagamento. As sementes de $H$. sucuuba oriundas dos ambientes de várzea e de terra firme não germinaram quando deixadas com o envoltório que as reveste, porém, entre aquelas nas quais o mesmo foi retirado, quase a totalidade germinou em um período máximo de 15 dias. Desta forma, é plausível supor que nesta espécie o envoltório constitui-se em barreira para a germinação. Tal situação pode ser verificada em muitas sementes de árvores tropicais, como por exemplo as do gênero Podocarpus e algumas espécies de Parkia (Kozlowski et al. 1991; Scarano \& Crawford 1992).

Embora as expansões do envoltório das sementes de $H$. sucuuba oriundas dos dois ambientes indiquem que a semente tem uma síndrome compatível com a dispersão anemocórica (Plumel 1991), a diferenciação entre essa síndrome e a hidrocórica é bastante difícil, uma vez que esta última freqüentemente utiliza caracteres que também servem aos propósitos da dispersão anemocórica. As duas apresentam em comum a forte conexão com um fator abiótico que envolve alto grau de casualidade e perda, baseando-se nas relações entre o peso e a área superficial da semente (Pijl 1972). Assim sendo, a mesma característica deve favorecer a dispersão da espécie pelo vento, particularmente nas áreas de terra firme, e pela água ou pela combinação de vento e água, nos ambientes de várzea. Dado o sucesso no estabelecimento da espécie nos dois ambientes, os experimentos de germinação realizados e as observações de campo feitas durante este estudo, propõe-se a expansão da classificação da planta de anemocórica, conforme postulado por Plumel (1991), para anemocórica e hidrocórica, uma vez que tanto o que foi observado no ambiente natural de várzea, quanto no experimento em viveiro, a semente de $H$. sucuuba pode permanecer horas flutuando até afundar por completo.

As características que determinam quando uma semente flutua ou afunda são muito importantes na sua dispersão e sobrevivência. De maneira geral, os mesmos envoltórios que facilitam a dispersão pelo vento ou pela água, constituem-se em barreiras

Tabela 1. Valores médios do peso de matéria seca (PS) e das medidas biométricas (comprimento, largura e espessura) das sementes de Himatanthus sucuuba Wood., oriundas dos ambientes de várzea e de terra firme $(\mathrm{n}=100)$.

\begin{tabular}{|c|c|c|c|c|c|c|c|}
\hline \multirow[t]{2}{*}{ Ambiente } & \multirow[t]{2}{*}{$\begin{array}{c}\text { PS } \\
\left(\mathrm{mg} \cdot \mathrm{g}^{-1} \mathrm{MS}\right)\end{array}$} & \multicolumn{2}{|c|}{$\begin{array}{l}\text { Comprimento } \\
(\mathrm{mm})\end{array}$} & \multicolumn{2}{|c|}{$\begin{array}{l}\text { Largura } \\
(\mathrm{mm})\end{array}$} & \multicolumn{2}{|c|}{$\begin{array}{l}\text { Espessura } \\
(\mathrm{mm})\end{array}$} \\
\hline & & com ala & sem ala & com ala & sem ala & com ala & sem ala \\
\hline Várzea & $12,76 \mathrm{a}$ & $61,80 \mathrm{a}$ & $19,22 \mathrm{a}$ & 51,20 a & $11,62 \mathrm{a}$ & $1,19 \mathrm{a}$ & $1,17 \mathrm{a}$ \\
\hline Terra Firme & $12,08 \mathrm{a}$ & 59,75 a & $20,01 \mathrm{a}$ & 52,06 a & $11,57 \mathrm{a}$ & $1,25 \mathrm{a}$ & $1,20 \mathrm{a}$ \\
\hline
\end{tabular}

Médias seguidas de mesma letra nas colunas, não diferem entre si pelo teste de Tukey a 5\% de probabilidade. 

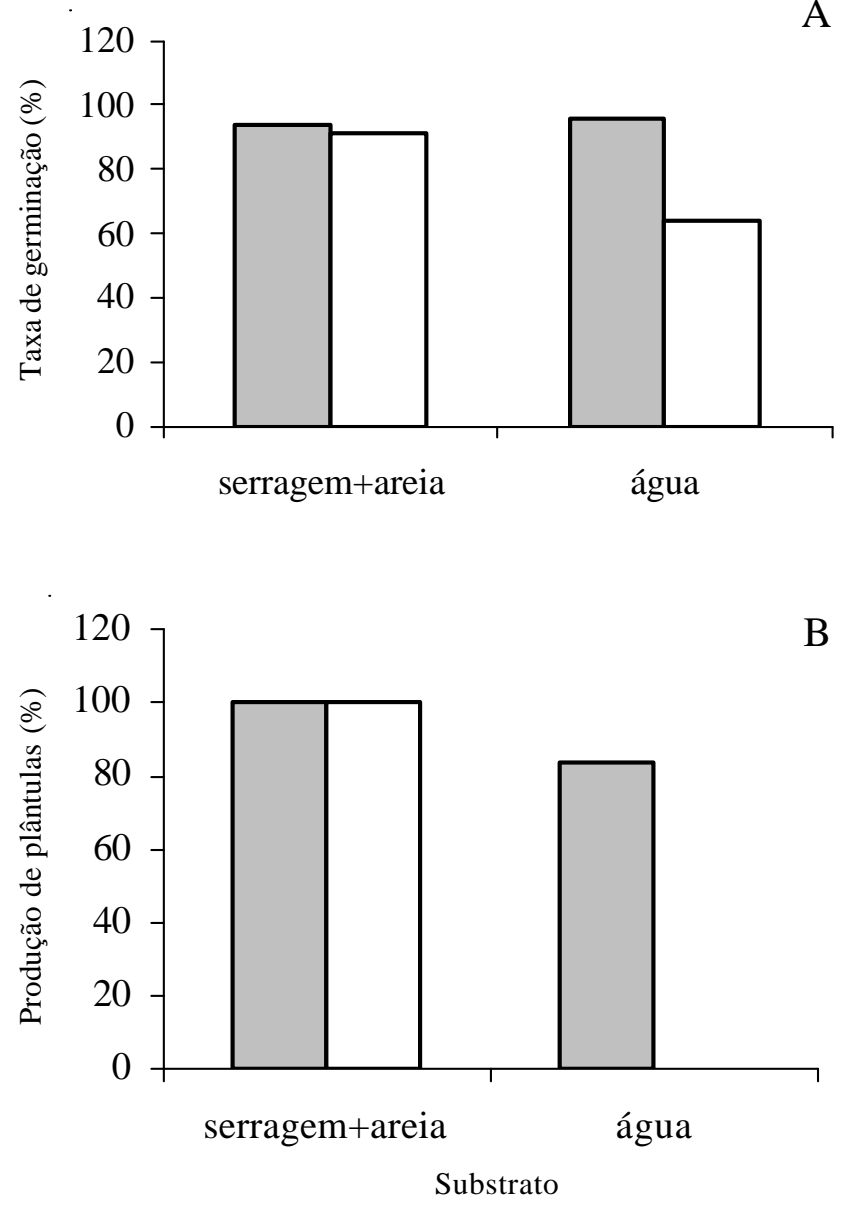

Figura 1. Média das taxas de germinação (A) e valores médios da produção de plântulas (B) de Himatanthus sucuuba Wood., dos ambientes de várzea e de terra firme, nos substratos areia+serragem ( $\mathrm{p}>0.5)$ e água $(\mathrm{p}<0.5),(\mathrm{n}=4)$. $\square=$ várzea; $\square=$ terra firme.

mecânicas que impõe dormência de forma tal que, em geral, a germinação não ocorre enquanto a semente estiver flutuando (Frankland et al. 1987). Outras espécies com revestimentos impermeáveis podem permanecer por períodos de vários meses sob submersão, antes de germinar, sem perder a viabilidade (Scarano 1998). O envoltório poderia também impor dormência em sementes intactas, por limitar a troca de gases para o embrião, particularmente o oxigênio (Bewley \& Black 1982).

O número de espécies que consegue germinar emitindo radícula ou caulículo sob anoxia é muito limitado (Crawford 1992). Dentre elas, várias espécies de gramíneas do gênero Oryza e Echinochloa podem germinar na total ausência de oxigênio, com taxas de germinação variáveis, conforme a espécie considerada. No entanto, a capacidade de germinar em condições anaeróbicas não está restrita às espécies aquáticas. Chorisia speciosa (A. St.-Hil.), árvore que habita a floresta de terra firme tropical brasileira, possui sementes que emitem radícula sob ausência total de oxigênio. Neste caso, a anoxia é condicionada pela presença de mucilagem nas sementes (Crawford 1992; Lobo \& Joly 1998). A germinação e o subsequiente crescimento de plântulas também podem ser verificados sob condições de hipoxia causada por alagamento, conforme foi observado em sementes de Inga affinis T.D. Penn e Sesbania virgata (Cav.) Pers. (Lobo \& Joly 1998).

Em H. sucuuba, uma vez removido o envoltório, a maioria das sementes oriundas dos dois ambientes (cerca de 90\%) germinou produzindo plântulas no tratamento não-alagado, onde as condições de oxigênio e temperatura foram em princípio ideais, contradizendo a postulação de Lorenzi (1998) que afirma baixa taxa de germinação para a espécie. Entretanto, quando a germinação foi feita na água, as sementes oriundas de ambientes de várzea permaneceram com altas taxas de germinação, produzindo plântulas saudáveis. A população oriunda da terra firme apresentou valor da taxa de germinação significativamente inferior, não chegando a produzir plântulas, uma vez que, após período máximo de cinco dias, ocorreu o apodrecimento da radícula. Este evento pode estar relacionado ao acúmulo de produtos tóxicos do metabolismo anaeróbico. C. Ferreira (dados não publicados) observou elevada atividade da enzima da via de fermentação alcoólica, álcool desidrogenase (ADH), em experimento posterior similar, porém que utilizava plântulas de H. sucuuba, sugerindo um desvio da via anaeróbica para a formação de etanol. Neste experimento, que teve a duração de 120 dias, as plântulas oriundas do ambiente de terra firme tiveram mortalidade de $100 \%$ antes do término das análises (ainda aos 90 dias), tendo apresentado como sintoma principal o apodrecimento do sistema radicular, seguido de morte da plântula. Por outro lado, as plântulas oriundas de ambientes de várzea apresentaram longevidade mesmo após o final do período experimental (C. Ferreira, dados não publicados), o que comumente é verificado em plantas consideradas não-tolerantes ao alagamento (Crawford 1992; Drew 1997; Kozlowski 1997).

Dessa forma, para $H$. sucuuba pode-se supor que a ação de mecanismos adaptativos envolvendo interações fisiológicas e metabólicas da mesma (Ferreira 2002) propiciam grande tolerância das populações oriundas dos dois ambientes ao alagamento, no que diz respeito à germinação. Entretanto, mesmo nesta, as populações dos dois ambientes já mostram diferenças significantes quanto aos percentuais de 
sucesso germinativo sob alagamento. Quando a análise considera o desenvolvimento das plântulas sob alagamento, as diferenças mostraram-se marcantes a ponto de sugerir algum grau de diferenciação das populações dos diferentes ambientes, merecedor de estudos posteriores.

\section{Agradecimentos}

Os autores são gratos à CAPES pela bolsa de fomento da MSc. Cristiane Ferreira; ao Instituto Nacional de Pesquisas da Amazônia, pelo aporte logístico; ao Projeto INPA/Max-Planck, PPI n. 1.31.50 e Projeto Shift de Produção Primária ENV-29, pelo suporte financeiro.

\section{Referências bibliográficas}

Bewley, J.D. \& Black, M. 1982. Viability, Dormancy and Environmental control. v.2. In: J.D. Bewley \& M. Black (eds.). Physiology and Biochemistry of seeds in relation to germination. New York, Springer-Verlag.

Brasil, 1992. Ministério da Agricultura e Reforma Agrária. Regras para Análises de Sementes. Brasília.

Campbell, D.G.; Daly, D.C.; Prance, G.T. \& Maciel, U.N. 1986. Quantitative ecological inventory of terra firme and várzea tropical forest on the Rio Xingu, Brazilian Amazon. Brittonia 38: 369-393.

Crawford, R.M.M. 1992. Oxygen availability as an ecological limit to plant distribution. Advances in Ecological Research 23: 93-185.

Drew, M.C. 1997. Oxygen deficiency and root metabolism: Injury and acclimation under hipoxia and anoxia. Annual Review of Plant Physiology. Plant Molecular Biology 48: 233-250.

Frankland, B.; Bartley, M.R. \& Spence, D.H.N. 1987. Germination under the water. Pp. 167-178. In: R.M.M. Crawford (ed.). Plant life in aquatic and amphibious habitats. Oxford, Blackwell.

Hook, D.D. 1984. Adaptations to flooding with fresh water. Pp. 265-294. In: T.T. Kozlowski (Ed.) Flooding and plant growth. New York, Academic Press.

Junk, W.J. 1989. Flood tolerance and tree distribution in Central Amazonian Floodplains. Pp. 47-64. In: L.B. Nielsen; I.C. Nielsen \& H. Baslev (eds.). Tropical forests: Botanical dynamics, speciation and diversity. London, Academic Press.

Kozlowski, T.T. 1997. Responses of woody plants to flooding and salinity. Tree Physiology Monograph, n. 1, p. 1-29.

Kozlowski, T.T. \& Pallardy, S.G. 1997. Growth control in woody plants. San Diego, Academic Press.

Kozlowski, T.T.; Kramer, P.J. \& Pallardy, S.G. 1991. The physiological ecology of woody plants. San Diego, Academic Press.
Lobo, P.C. \& Joly, C.A. 1998. Tolerance to hypoxia and anoxia in Neotropical tree species. Oecologia Brasiliensis 4: 137-156.

Lorenzi, H. 1998. Árvores brasileiras: manual de identificação e cultivo de plantas arbóreas nativas do Brasil. $2^{\mathrm{a}}$ ed. Nova Odessa, São Paulo, Plantarum.

Parolin, P. 2000. Phenology and $\mathrm{CO}_{2}$-assimilation of trees in Central Amazonian floodplains. Journal of Tropical Ecology 16(3): 465-473.

Parolin, P. 2001. Seed germination and early establishment of 12 tree species from nutrient-rich and nutrient-poor Central Amazonian floodplains. Aquatic Botany 70: 89-103.

Piedade, M.T.F.; Junk, W.W. \& Parolin, P. 2000. The flood pulse and photosynthetic response of trees in a white water floodplain (várzea) of Central Amazon, Brazil. Verhandlungen der Internationale Vereinigung für Limnologie 27: 1734-1739.

Piedade, M.T.F.; Worbes, M. \& Junk, W.J. 2001. Geoecological controls on elemental fluxes in communities of higher plants in Amazonian floodplains. In The Biogeochemistry of the Amazon Basin. Pp. 209-234. (M.E. McClain; R.L.Victoria \& J.E. Richey, eds.). New York, Oxford University Press.

Pijl, L. van der. 1972. Principles of dispersal in higher plants. $2^{\text {nd }}$ ed. Berlin, Springer-Verlag.

Plumel, M.M. 1991. Le genre Himatanthus (Apocynaceae). Révision taxonomique. Bradea 5: 1-118.

Ribeiro, J.E.L.S.; Hopkins, M.J.G.; Vicentini, A.; Sothers, C.A.; Costa, M.A.S.; Brito, J.M.; Souza, M.A.D.; Martins, L.H.P.; Lohmann, L.G.; Assunção, P.A.C.L.; Pereira, E.C.; Silva, C.F.; Mesquita, M.R. \& Procópio, L.C. 1999. Flora da Reserva Ducke - Guia de identificação das plantas vasculares de uma floresta de terra-firme na Amazônia Central. Manaus, INPA.

Scarano, F.R. 1998. A comparison of dispersal, germination and establishment of woody plants subjected to distinct flooding regimes in Brazilian flood-prone forests and estuarine vegetation. Pp. 177-193. In: F.R. Scarano \& A.C. Franco (eds.). Ecophysiological strategies of xerophytic and amphibious plants in the neotropics. Series Oecologia Brasiliensis v. IV. Rio de Janeiro, PPGE, Universidade Federal do Rio de Janeiro.

Scarano, F.R. \& Crawford, R.M.M. 1992. Ontogeny and the concept of anoxia-tolerance: the case of the Amazonian leguminous tree Parkia pendula. Journal of Tropical Ecology 8: 349-352.

Villegas, L.F.; Fernández, I.D.; Maldonado, H.; Torres, R.; Zavaleta, A.; Vaisberg, A.J. \& Hammond, G.B. 1997. Evaluation of the wound-healing activity of selected traditional medicinal plants from Perú. Journal of Ethnopharmacology 55: 193-200.

Wittmann, F.; Anhuf, D. \& Junk. W.J. 2002. Tree species distribution and community structure of central Amazonian várzea forests by remote-sensing techniques. Journal of Tropical Ecology. 18: 805-820. 
